\title{
(2) OPEN ACCESS \\ New life for old cellular pathology: a transformational approach to the upcycling of historic e-pathology records for contemporary clinical uses
}

\author{
David Anthony Rew 지, ${ }^{1}$ Alan Arthur Hales, ${ }^{2}$ David Cable, ${ }^{2}$ Keith Burrill, ${ }^{3}$ \\ Adrian C Bateman (i) ${ }^{3}$
}

${ }^{1}$ Faculty of Medicine, University of Southampton, Southampton, UK

${ }^{2}$ Information Technology, University Hospital Southampton NHS Foundation Trust, Southampton, UK ${ }^{3}$ Cellular Pathology, University Hospital Southampton NHS Foundation Trust, Southampton, UK

\section{Correspondence to}

David Anthony Rew, Faculty of Medicine, University of Southampton, Southampton S0166YD, UK; dr1@soton. ac.uk

Received 3 January 2021 Revised 10 January 2021 Accepted 12 January 2021
Check for updates

(c) Author(s) (or their employer(s)) 2021. Re-use permitted under CC BY-NC. No commercial re-use. See rights and permissions. Published by BMJ.

To cite: Rew DA, Hales AA, Cable D, et al. J Clin Pathol Epub ahead of print:

[please include Day Month

Year]. doi:10.1136

jclinpath-2021-207385

\section{ABSTRACT}

Aims Cellular pathology ('e-pathology') record sets are a rich data resource with which to populate the electronic patient record (EPR). Accessible reports, even decades old, can be of great value in contemporary clinical decision making and as a resource for longitudinal clinical research. The aim of this short paper is to describe a solution in a major UK University Hospital which gives immediate visibility and clinical utility to 30 years of e-pathology records

Methods Over the past decade, we have created a timeline structured and iconographic data framework for the 'whole-of-life' visualisation of the entirety of an EPR. We have enhanced this interface with the sequential extraction of 373342 e-pathology reports from legacy Ferranti (1990-1997) and Masterlab (1997-2004) files. They have been uploaded into our SQL file servers, following appropriate data quality and patient identity reconciliation checks.

Results We have restored a large repository of previously inaccessible e-pathology records to clinical use and to immediacy of access as a foundation element of our timeline structured EPR. This process has also allowed us to populate and validate an EPR-integral breast cancer data system of 20000 cases with epathology records dating back to 1990 .

Conclusions The revitalisation of old e-pathology reports into a timeline structured EPR creates preserves and upcycles the investment in pathology reporting which is otherwise progressively lost to clinical use. Epathology records provide reliable, life-long evidence of critical transition points in individual lives and disease progression for clinical and research use, when they can be instantly accessed.

\section{INTRODUCTION}

Cellular pathology ('e-pathology') reports of biopsies and operative specimens provide a rich record of key clinical events in an individual's life. They document the diagnosis or exclusion of a disease: the specifics of that disease; the place, time and nature of the procedure that generated the sample for analysis; and by whom the specimen was generated and reported. As such, they are key anchor and reference points in the patient's clinical record, whether in paper files or digital systems.

Legacy filing systems inevitably create slippage in the accessibility of a pathology record. Printed reports are commonly overlain by more recent documents and back-catalogued in older storage files and bulky clinical record folders. Electronic files drop down document search lists and then out of sight as legacy computer systems are replaced by newer systems, running on different code from different suppliers.

Modern digital systems permit the presentation of clinical data in ways which are only limited by the imagination. However, many digital pathology record systems rely on legacy interface designs and functionality. These oblige busy health professionals or researchers to sift through multiple software systems, pages, tabs, frames, lists and files to recreate the electronic patient record (EPR), at a substantial cost of resource.

The importance of the retention and storage of pathological records and archives has long been recognised by the UK's Royal College of Pathologists and by the Institute of Biomedical Science, since the first edition of guidelines was published in 1994. Record storage and archiving policies have been widely adopted and implemented across the UK National Health Service (NHS).

Cellular pathology reports were among the first to be digitised in the UK's national transition from paper-based to digital health data systems in the early 1990s. Early systems were stand-alone, with unique and clunky code and challenging data extraction routines. Although valuable in their time, these systems were not designed for integration into the modern EPR and files were effectively and functionally lost as legacy computer systems were replaced.

In a paper on 'Archiving the Phenome' in 2009, Milton Corn noted that clinical records deserve long-term preservation and he asked 'how valuable really is old clinical information?'. Based on US experience and while his focus was not specifically on pathology records, he noted with foresight that 'the somewhat whimsical treatment of paper records in office practice will probably carry over to electronic records as office Electronic Health Records (EHRs) become more common, unless policies and policy enforcement change distinctly'.

Three decades of digital revolution since 1990 have created a rich internet resource on archiving policies and on the accurate and immediate dissemination and storage of e-pathology results. However, the global literature and internet resources have almost nothing to say on the retrieval and display of old cellular pathology reports, as the natural corollary of their archiving and storage. 


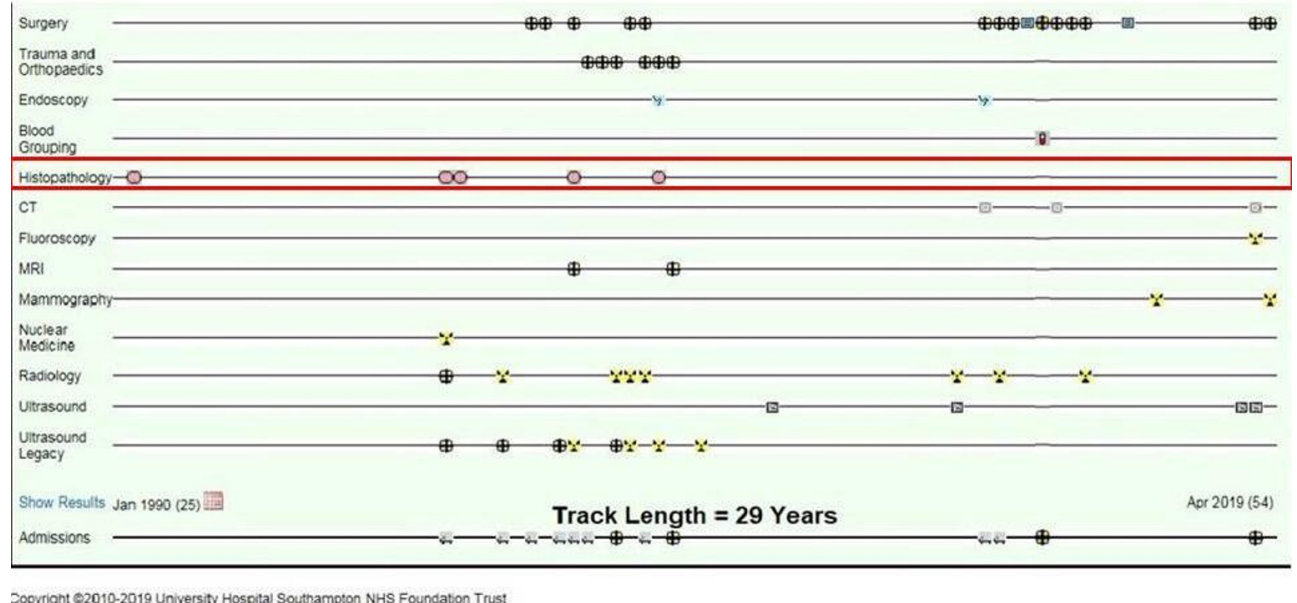

Figure 1 This is a live screenshot of the University Hospital Southampton Lifelines interface in its 2019 iteration. It displays the e-records of a Spina Bifida patient with complex medical needs. The histopathology timeline (outlined in red) is a central element of the framework, as the histopathology record icons (the pink disc icons) provide critical orientation and reference points for other imaging reports and clinical documents. Note that the earliest e-pathology report is in early 1990. Electronic documentation otherwise started around 1995, before which all other records were on paper so they are not displayed electronically.

Over the past decade, we have developed a radical approach to the surfacing of the electronic documents, reports and summaries which underpin the individual EPR.

We have created a data framework of stacked, synchronised and subject-specific timelines and dynamic iconographic representation of documents, reports and other clinical events. ${ }^{2}$ This methodology permits the interactive display of the entire 'wholeof-life' digital record set for any patient on a single screen.

This format provides context and immediacy of access to any record of any age. It allows a clinician to read the detail of a record of any age with a single mouse click and it permits a range of agile clinical research strategies on large cohorts of patients, which would otherwise be impractical with legacy paper and older digital record systems.

In 2010, early in the development of this interface, we recognised that the integration of legacy electronic cellular pathology records would transform the utility both of the interface and of the e-pathology records themselves. Here, we describe our experience with this approach and the benefits that should flow from wider adoption of the approach.

\section{METHODS}

University Hospital Southampton (UHS) Lifelines is an htmlenabled system whose framework code was progressively written in Microsoft ASP code from 2010 to 2015, when it was converted to MS Dot Net Code. UHS Lifelines is integrated into the UHS clinical data estate, such that all e-pathology reports are directly loaded into our proprietary e-Quest Laboratory results reporting system.

From there they are pulled directly into the UHS Lifelines EPR and loaded onto the e-pathology timeline as dynamic icons at the relevant time point (figure 1). The icons link directly to the relevant reports. Hovering over an e-pathology icon displays the relevant metadata, while clicking on the icon opens the report.

The reports are all presented as analogue documents which are unchanged from the original electronically recorded text or image, so the quality of the report is entirely as generated by the original reporting pathologist.

Discussions within our group began in 2010 as to the likely accessible historic e-pathology record holdings and their formatting. Prior to 1990, records were held on a card index and a microfilm system. While it would have been possible to digitise these images and add metadata, this would have been a resource intensive exercise which was not justifiable, not least as reporting standards prior to 1990 rendered many of the cancer reports of limited value.

\section{Sourcing our e-pathology reports}

UHS pathology reports were originally written and stored in a Ferranti Computer System from January 1990 to mid-1997. These were transferred to the Clinisys (previously Masterlab) Labcentre system when the UHS Laboratory Information System (LIMS) went live in 1997.

We ran queries on our systems which demonstrated that histopathology (HI) and neuropathology (NE) reports were available in Labcentre in the LMI.WP.REPORT format from June 1997 to April 2003.

A further date range search from May 1997 back to April 1990 on the HI and NE files on Labcentre uncovered records in the LMI.RESULT.RQS format which had been imported from the Ferranti system.

\section{Data validation}

Once the queries for the histology reports were complete, we undertook a data validation exercise to link patient identifiers between our Patient Master Index (PMI) and those on the individual reports. To be valid, the hospital number, NHS number and demographic data of any subject patient had to match the data exported with the ex-Ferranti data.

In the non-matching reports, the patient validation ranged from a minor mismatch to complete non-matches (ie, no patient on the PMI bearing any similar attributes). The data cleansing of these reports would have been manually intensive and often very difficult, so these reports were excluded from further consideration.

\section{Live uploading of historic data}

During 2013 and 2014, 373342 HI reports were uploaded from the Ferranti and Masterlab/Labcentre system into the Trust's EQuest EPR results reporting system, which feeds UHS Lifelines with data. 
We felt that this was best result that could be achieved with limited resources between daily backups from our live LIMS. Of note is that our laboratory now has a more efficient and user friendly Business Objects tool (Sage Software) for pathology record extraction from our data warehouse, from June 2013 onwards.

We then traced and uploaded all traceable e-cytopathology reports, excluding cervical cytology reports which the hospital no longer processed.

Each e-report had been historically tagged with sufficient metadata, in the form of a date/time stamp and its identity, to permit the UHS Lifelines software to allocate the report a place in two-dimensional time and space (the subject timeline) on the EPR interface without further modification.

\section{RESULTS}

Following the uploading process, all current and the back-loaded legacy cellular pathology reports are now available for immediate recall and real time display whenever an individual EPR is called up in the UHS Lifelines format. New reports are immediately added to the live database and are displayable as soon as they are signed off. There is no significant latency in the display of the 'cellular pathology' timeline within UHS Lifelines.

\section{Evidence of practical impact}

\section{Anecdotal and experiential}

The utility of immediacy of access to historic cellular pathology records is both anecdotal and experiential. Our own hospital has moved rapidly to the abolition of legacy paper records and files from outpatient and inpatient services. Therefore, if data do not exist electronically, then they are effectively out of circulation. Such old paper files as are retained are often held in offsite record repositories where recall other than for major investigational purposes is wholly impractical. Our bespoke solution overcomes this problem. For example, one surgical user of UHS Lifelines reported that:

in a recent outpatient consultation, a patient had been referred with a recurrent goitre following earlier surgery in the district in 1996.

The old operating records had been lost, but I was immediately able to locate and understand the fact, date and the type of the earlier thyroid surgery directly from the 1996 histopathology report.

\section{Breast cancer data system exemplar}

The Southampton Breast Cancer Data System (SBCDS) contains $20000+$ 'whole of life' records of patients who have been treated locally since our card index records began in the 1970s, whereas electronic documents with time and source metadata were first systematically created in our UHS clinical data estate in the mid-1990s.

SBCDS is founded on a master Lifetrack which describes the progression of breast neoplasia from first diagnosis, via locoregional recurrence to the appearance of distant metastases and final outcome. We are also able to record synchronous and metachronous morbid cancers of other types on the timeline (eg, gynaecological, colorectal, lung, lymphoma) for clinical visibility and future research purposes (figure 2).

It incorporates UHS Lifelines into every patient data entry screen, thus integrating the continuously updated evidence base directly into the record.

The upcycling of early e-pathology records from January 1990 onwards provided a very substantial uplift to our data system. Following the 1990-1997 backload, 6942 e-histology (ex-Ferranti) reports of all types could be directly linked to 4021 patient records, including many which reported on breast tissue and cancer specimens.

This high match rate made a substantial contribution to the validation of the historic SBCDS records between 1990 and 1997. In effect, it extended our starting point for e-records back to 1990 , coinciding with the origins of the national breast screening programme.

\section{DISCUSSION}

Cellular pathology records provide reliable, consistent and validated documentation of critical transition points in individual lives and disease progression. They retain fundamental clinical and research value long after their immediate clinical utility has passed, as long as they can be readily accessed. We have

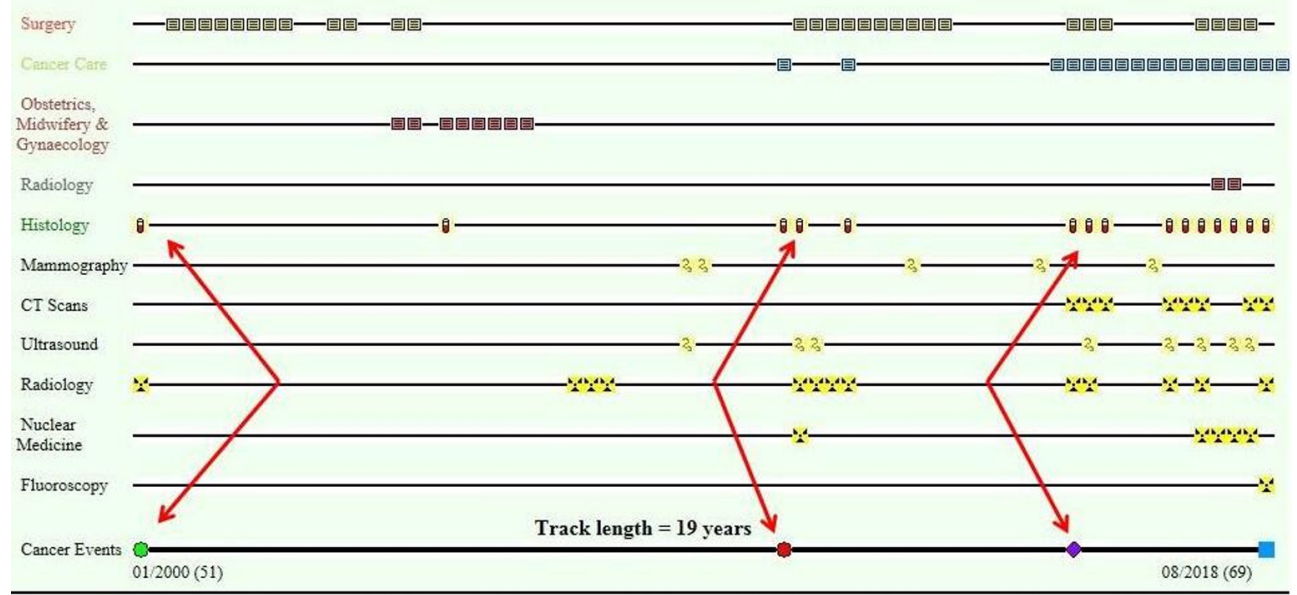

Figure 2 A screenshot of the electronic patient record (EPR) of a single patient in the Southampton Breast Cancer Data System, as visualised on an early development version of the University Hospital Southampton Lifelines EPR interface, within which cellular pathology records are each represented and accessed via the cylindrical icon. This illustrates the utility of 'historic' pathology records. Each pair of red arrows highlights the linkage between a cancer event and the confirmatory histology report. This particular patient developed a right sided breast neoplasm (green circle) in 2000 at the age of 51; a left sided breast neoplasm in 2011 (red circle); and chronic lymphocytic leukaemia (purple diamond marker) in 2016, from which she subsequently died aged 69. Clicking on each icon in the live system opens the relevant document or report. 
demonstrated how new life and utility can be brought to electronic cellular pathology records of any age, through ${ }^{1}$ importation of the original data files into a modern database ${ }^{2}$; linking directly into the EPR and specifically into a data visualisation interface and ${ }^{3}$ creating access to those files in an appropriate retrieval and visualisation system.

\section{Post hoc utility of pathology reports}

Pathology reports represent a huge investment in professional time, intellectual effort and technical resources across the pathology community. In terms of information value, pathology reports a 'gold standard' in the diagnosis and progression of many diseases. Cellular pathology reports may also provide information about the operation from which the specimens are sourced. Cellular pathology has its greatest utility in the immediate determination of diagnoses and treatment guidance and often also predicts outcomes for patients with cancer.

\section{Significance of timeline structured visualisation of the EPR}

The digital transformation of global health services is still at an early stage. Our interactive, timeline structured and iconographic EPR interface represents a radical, agile and iterative experiment in clinical data visualisation and interaction which is still in evolution.

Nevertheless, the ability to visualise an entire EPR on a single screen has profound consequences for the clinical user. In the context of legacy e-records of any age, it transforms the "here, now and in the very recent past' interaction with clinical and pathology records, into a 'whole of life' or 'whole of disease process' interaction.

Moreover, electronic records of any age have the same immediacy of access as contemporary reports and documents. This 'immediate availability' of pathology records in a few mouse clicks has very significant consequences for the clinical utility of the overall EPR, because the need to search through old files and indices for individual e-pathology records is abolished. As we have shown, benefits are that:

1. This approach finds applications in daily clinical activity at any point of contact between clinicians, outpatients and inpatients where reference needs to be made to the clinical history.

2. It facilitates the ease and speed of review of cohorts of records for clinical research projects.

3. It permits new approaches to clinical research, including the validation of timeline structured descriptive and data models of disease progression.

\section{Utility of our data visualisation model to pathologists}

Cellular pathologists are often faced with the challenge of contextualising a laboratory specimen within the clinical history. The universal availability of the UHS Lifelines interface to authorised users of our system permits ease of access to the individual EPR and hence also to the pathology history in the cut up room, reporting room or the postmortem laboratory.

\section{Constraints on wider adoption of the Southampton EPR interface format}

The UHS lifelines interface is a bespoke product of the unique, locally owned and developed Southampton clinical data estate, which permits extensive experimentation and IT product roll out on our own servers.

We believe it to be a very powerful tool for clinical use, through the radical simplification of the human-machine interactions around clinical data. Such opportunities may not be so readily available in other health organisations. We recognise that in its present form, the UHS Lifelines system is confined to locally generated and stored electronic records which are traceable, retrievable and uploadable from across our own clinical data estate.

The concept is universal in its healthcare record applications, but the code is not exportable to the widely used EPR systems of the major commercial providers. Users and their national representative bodies will therefore need to develop their requirements and negotiate their cases with other software providers.

In respect of the historic pathology data, we have been constrained by the availability of those records in historic format and by our ability to match the old e-records to other patient records in the hospital's PMI. Coverage of this archive has not been complete but it has nevertheless substantially enriched our visual EPR.

Of course, many of the older HI records apply to patients who have since died. These records nevertheless have particular value for clinical research projects, as evidenced in the accurate population and validation of the SBCDS.

\section{Issues around the whole of life retention of cellular pathology records}

This leads us into the interesting and unresolved question as to how long clinically relevant records, including HI records, should be retained after their generation and after death, now that digital storage is so much easier than the storage of paper records.

In the 2015 guidelines from the Royal College of Pathologists and the Institute of Biomedical Sciences, ${ }^{3}$ the recommendation was that electronic or hard copies of reports should be kept for at least 30 years by the laboratory, with maintained accessibility of e-copies when laboratory computer systems are upgraded or replaced.

Our specific experience in extending the longevity of e-records would argue for a 'whole of life' retention of such records, extending after death, to facilitate long-term clinical research, including the application of predictive analytics and tools of artificial intelligence to help better understand disease processes.

This prompts question of whether postmortem examination reports should be uploaded to the EPR. This is technically feasible and there may also be long-term research benefit from the rich evidence of actual outcomes and causation which are contained in postmortem examination reports.

The Royal College of Pathologists guidelines state broadly that

'post-mortem reports should be lodged in patient's record. In the case of Coroner's or Fiscal's reports, this depends upon the Coroner's or Fiscal's approval. Electronic or hard copy should be kept for at least eightyears with maintained accessibility. In cases of violent or suspicious death, post-mortem reports should be retained for 30 years, with continuation of access to e-copies when laboratory computer systems are upgraded or replaced. This guidance applies equally to rapid, short reports that may be prepared for the Coroner, summarising cause of death, and to the final reports of post-mortem examinations'.

There is scope for examination of these matters as new approaches to the digital EPR, such as exemplified in our work on UHS Lifelines, become mainstream.

Therefore, in conclusion, we have sought to demonstrate that e-pathology reports have a utility in 'whole of life' clinical record keeping, which extends well beyond their immediate clinical utility. 
We propose that new formats for the display of clinical information within the interactive EPR should now prompt a systemwide examination of the 'whole-of-life' utility of e-pathology records of all eras in the modern and evolving EPR.

\section{Take home messages}

- Cellular pathology records have lifelong clinical utility.

- Historic electronic cellular pathology records with appropriate metadata can be readily upcycled in bulk into modern electronic patient record (EPR) systems.

- Uploading of cellular pathology e-records into 'next generation' timeline structured EPR interfaces, such as exemplified by the University Hospital Southampton Lifelines system, allows their immediate visualisation and contextualisation as far back as 1990.

- The upcycling of electronic records adds enduring value to the substantial costs of creating reports and providing cellular pathology services.

- These practical observations should prompt the reexamination of clinical record storage policies in favour of 'whole of life' systems.

Handling editor Runjan Chetty.

Contributors DAR conceived the project and wrote the manuscript. KB and $A C B$ sourced and supplied the pathology e- records. AAH and DC managed the integration of the e-records into the UHS EPR, including quality control and data validation procedures.

Funding The authors have not declared a specific grant for this research from any funding agency in the public, commercial or not-for-profit sectors.

Competing interests None declared.

Patient consent for publication Not required.

Provenance and peer review Not commissioned; internally peer reviewed.

Data availability statement Data sharing not applicable as no datasets generated and/or analysed for this study.

Open access This is an open access article distributed in accordance with the Creative Commons Attribution Non Commercial (CC BY-NC 4.0) license, which permits others to distribute, remix, adapt, build upon this work non-commercially, and license their derivative works on different terms, provided the original work is properly cited, appropriate credit is given, any changes made indicated, and the use is non-commercial. See: http://creativecommons.org/licenses/by-nc/4.0/.

\section{ORCID iDs}

David Anthony Rew http://orcid.org/0000-0002-4518-2667

Adrian C Bateman http://orcid.org/0000-0003-2222-4104

\section{REFERENCES}

1 Corn M. Archiving the phenome: clinical records deserve long-term preservation. J Am Med Inform Assoc 2009;16:1-6.

2 Hales AA, Cable D, Crossley E, et al. Design and implementation of the stacked, synchronised and iconographic timeline-structured electronic patient record in a UK NHS global digital exemplar Hospital. BMJ Health Care Inform 2019;26:e100025.

3 Royal College of Pathologists. The retention and storage of pathological records and specimens (5th edition), 2015. Available: https://www.rcpath.org/resourcelibrary/theretention-and-storage-of-pathological-records-and-specimens-5th-edition-html 